\title{
Robotic Path Planning for Surgeon Skill Evaluation in Minimally-Invasive Sinus Surgery
}

\author{
Narges Ahmidi ${ }^{1}$, Gregory D. Hager ${ }^{1}$, Lisa Ishii ${ }^{2}$, Gary L. Gallia ${ }^{3}$, and Masaru Ishii ${ }^{2}$ \\ ${ }^{1}$ Department of Computer Science, Johns Hopkins University, Baltimore, MD 21218 \\ nahmidi1@jhu.edu, hager@cs.jhu.edu \\ ${ }^{2}$ Department of Otolaryngology-Head \& Neck Surgery, \\ Johns Hopkins School of Medicine, Baltimore, MD 21287 \\ \{learnes2, mishii3\}@jhmi.edu \\ ${ }^{3}$ Department of Neurosurgery, Johns Hopkins University School of Medicine, \\ Baltimore, MD 21287 \\ ggallial@jhmi.edu
}

\begin{abstract}
We observe that expert surgeons performing MIS learn to minimize their tool path length and avoid collisions with vital structures. We thus conjecture that an expert surgeon's tool paths can be predicted by minimizing an appropriate energy function. We hypothesize that this reference path will be closer to an expert with greater skill, as measured by an objective measurement instrument such as Objective Structured Assessment of Technical Skill (OSATS).

To test this hypothesis, we have developed a Surgical Path Planner (SPP) for Functional Endoscopic Sinus Surgery (FESS). We measure the similarity between an automatically generated reference path and surgical motions of subjects. We also develop a complementary similarity metric by translating tool motion to a coordinate-independent coding of motion, which we call the Descriptive Curve Coding (DCC) method.

We evaluate our methods on surgical motions recorded from FESS training tasks. The results show that the SPP reference path predicts the OSATS scores with $88 \%$ accuracy. We also show that motions coded with DCC predict OSATS scores with $90 \%$ accuracy. Finally, the combination of SPP and DCC identifies surgical skill with $93 \%$ accuracy.
\end{abstract}

Keywords: Robotic path planning, Skill evaluation, Motions curvature representation, Support Vector Machine, OSATS, Minimally invasive surgery.

\section{Introduction}

Hand-eye coordination is a crucial skill in Minimally-Invasive Surgeries (MIS). This is particularly true for Functional Endoscopic Sinus Surgeries (FESS), where surgeon performance is limited by indirect observation and highly constrained tool movements inside the sinus cavity. For surgeons in training, their overall expertise level is expected to rise (from novice to expert), but their actual skill at a given point in time may vary widely between subjects, between tasks and also between task executions (trials). Thus, time and experience are not necessarily predictors of skill. An alternative is Objective 
Structured Assessment of Technical Skills (OSATS) [1] where a committee of faculty surgeons ranks residents' surgical trials.

Past work on the automation of surgical Table 1. The relationship between self-declared scores (Expert or Novice) and the corresponding OSATS scores (Level-3 is expert and level-0 is novice).

\begin{tabular}{|c|l|l|l|l|l|}
\cline { 3 - 6 } \multicolumn{2}{c|}{} & \multicolumn{4}{c|}{ OSATS scores } \\
\cline { 3 - 6 } \multicolumn{2}{c|}{} & Level-0 & Level-1 & Level-2 & Level-3 \\
\hline Self-declared scores & Expert & $5 \%$ & $6 \%$ & $10 \%$ & $79 \%$ \\
\cline { 2 - 6 } & Novice & $45 \%$ & $20 \%$ & $19 \%$ & $16 \%$ \\
\hline
\end{tabular}

skill assessment has primarily relied on self-declared skill [2] mainly using techniques based on Hidden Markov Models (HMM) [2-5]. But, is self-declared skill consistent with OSATS? To answer this question, we ran an experiment by asking two independent groups of faculty surgeons to grade two MIS datasets (da Vinci robotic surgery and FESS) based on OSATS (level-0 to 3). We grouped levels 0 and 1 as Novices and levels 2 and 3 as Experts and then calculated the mismatch rate between the OSATS and self-declared scores $(25 \%$ of the time with p-value $<0.0001$ ) (Table 1).

We hypothesize that, most of the differentiation in OSATS scores arises from the complexity in manipulating tools in MIS (e.g. avoiding critical tissues and finding the correct passage). Thus, a plausible approach to OSATS evaluation for MIS may be to first compute an optimal path, and then to compare measured paths to them. The idea of motion planning for MIS procedures is not new [6-8], but there is no prior study on using these paths as a basis for skill assessment.

In this paper, we address the problem of automated OSATS evaluation of skill by exploring the following two questions: (1) Can comparison with an optimal surgical path be used to establish a measure for skill evaluation? and (2) Does quality of motion, independent of path, provide additional information? To answer these questions we develop a Surgical Path Planner (SPP) and measure its similarity to recorded data from surgeons in training. To improve the similarity metric, we introduce the Descriptive Curve Coding (DCC) method which translates motion from Cartesian space into a coded string. This DCC string efficiently describes surgeon motion in a way that amplifies differences due to skill. We evaluate both SPP and DCC on the FESS trials, and report the similarity of the measured scores with respect to the OSATS scores.

\section{Experimental Setup}

A group of 20 subjects participated in the data collection (self-declared: 7 experts and 13 novices). A nasal surgery simulator was developed for this study, which consisted of a partially dissected cadaver head, a high-resolution video tower, tracked sinus endoscopes and a straight tracked pointer. A calibration process is then performed to find the tool tip location from the initial location of the sensors attached to the outer end of the tools. Subjects were naïve to the nasal anatomy and performed the tasks in a random and untimed fashion. Each surgeon performed 3 different non-destructive tasks repeatedly over a two-day period. Test subjects were allowed to familiarize themselves with the data collection setup prior to the data collection process and care 
was taken to simulate the surgical environment typically encountered in an operating room as best as possible.

The targets were: right Maxillary Sinus (MS.r), right Eustachian Tube (ET.r), and left Lamina Papyracea (LP.1). The tool motions were recorded using an electromagnetic tracker (Fig.1) from a total of 109 trials: 36 MS.r (19 Expert/17 Novice), 34 ET.r (20/14), 39 LP.1 (24/15). Three supervising faculty surgeons independently scored each trial using two-level grades ("expert/1" and "novice/0") and the total of their scores is used as the 4-level OSATS score (0 to 3 ).

For the purposes of analysis, the recorded surgical motions are transformed to the CT coordinate system using a landmark-based rigid registration.

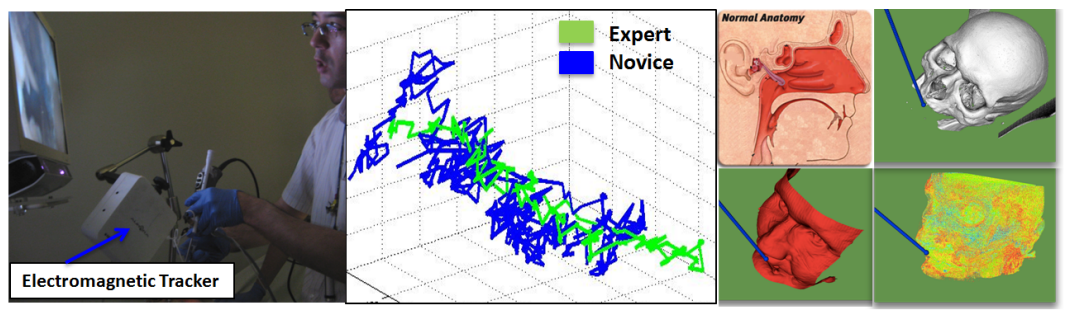

Fig. 1. (Left) Experiment setup. (Middle) Sample of tool motion in the Cartesian-space (Right) Sinus anatomy the bones/skin/soft tissue extracted from CT (blue line is the endoscopic)

\section{Methodology}

In this section, we introduce two methods: the Surgical Path Planner and the Descriptive Curve Coder. We then define how they are used to replicate OSATS scores (Fig. 2). Additionally, an OSATS-based HMM model is implemented using the algorithm reported in [4] on our dataset.

Surgical Path Planner (SPP): The nose is compact and contains many structures. Some of these structures like the middle and superior turbinate are involved in olfaction and are thus optimized to maximize surface area. This leaves few regions within the nose that are freely accessible with ballistic, collision-free paths; therefore a surgeon has to displace tissues to access many parts of the nose with a straight instrument. Our algorithm generates a surgical path optimized primarily to find the shortest path between the nostril and the target. It secondarily minimizes the number and extent of tissue-instrument collisions. Nasal anatomy was extracted from a CT of the paranasal sinuses. The stiffness of the obstacles is defined by the intensity of the CT voxels separated into 3 types: skin, bones and soft tissue. The endoscopic tool is modeled as a cylindrical robot of length $200 \mathrm{~mm}$, radius $2 \mathrm{~mm}$ with a $5 \mathrm{DoF}$ (degrees of freedom) with pose $H$ in the CT coordinate system. The reference path (a sequence of $H^{t}$ from start to goal over time $t$ ) is calculated by the following two steps:

(1) Probabilistic Road Map (PRM): Given the start and the goal point, we solve for the 3D translation motion of the tool tip using a PRM algorithm [9] with a uniform distribution. Dijkstra's algorithm then finds the shortest path toward the goal in the 3D Cartesian free-space. This initial path is used for the second step where we solve for the remaining $2 \mathrm{DoF}$ rotational motions of the robot. 


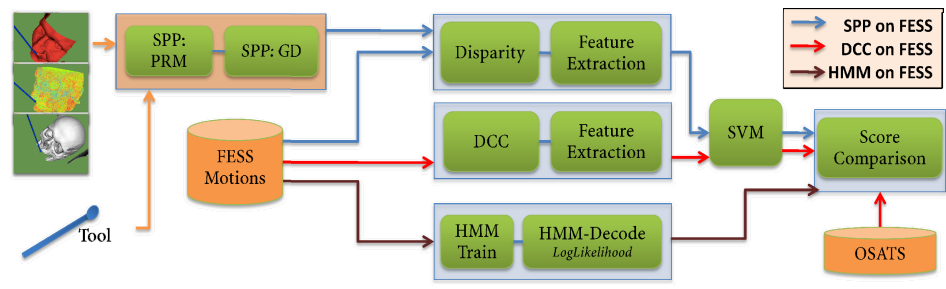

Fig. 2. The system components and the data flow for the SPP and DCC methods

(2) Gradient Descent: The robot starts to follow the initial path toward the goal (with an initial straight-to-goal orientation). If a collision happens, a numerical gradient descent approach [9] updates the robot orientation (the rotational components of $H^{t}$ ) by minimizing the collision cost function $\mathcal{F}$ (with higher/lower $\gamma_{\mathrm{j}}$ values for collision with bones/soft tissue, respectively):

$$
\mathcal{F}\left(H^{t}\right)=\sum_{j=\{\text { skin,softtissue,bones }\}} \gamma_{j} * \operatorname{Collision}_{j}\left(H^{t}\right)
$$

where Collision is measuring the overlap of the robot with the $j$ th CT layers. The minimization approach calculates the gradient of the repulsive forces $\alpha$ on the robot. Using the normalized $\alpha$, the robot then moves away from the obstacles toward the position with the least collision with the tissue and updates its location:

$$
H^{t+1}=H_{\alpha}^{t}=H^{t} * H_{a_{\varphi}}^{t} * H_{\alpha_{\theta}}^{t} * H_{\alpha_{x}}^{t} * H_{\alpha_{y}}^{t} * H_{\alpha_{z}}^{t}
$$

This procedure continues until the robot reaches the goal. To measure the disparity between the generated SPP path and each surgical trial, we measure the distance between each point on the surgical motion to its closest neighbor on the SPP path in Cartesian space. For each trial, we generate a feature vector containing the pairwise correlation between SPP path and the surgical path axes as well as the mean and the standard deviation of their disparities. Using these 11 dimension (9: correlation, 1: mean, 1: std) feature vectors, a Support Vector Machine (SVM) is trained (leave-one trial-out for test) to classify surgical trials to 4-classes (OSATS level for FESS). The expertise level of each trial is defined by its corresponding class label.

Descriptive Curve Coding (DCC): A drawback for skill evaluation methods based on Cartesian motions is that they do not describe the "texture" of the motion - i.e. coarseness, loops, sudden change of direction etc. - which appears to be highly correlated with skill. Hence, we introduce a new method for describing the geometrical curvature of a given 3D motion and investigate its success in skill evaluation. The idea of representing 3D curves based on their curvature has been heavily studied. For example, Frenet-Serret orthonormal frames [10] represent torsion and curvature of a non-degenerate curve, whereas authors in [11] measure the dissimilarity between two discrete curves by defining an orthogonal directional chain code. However, these methods were never used to represent surgical motions; we believe they can serve as an efficient vocabulary for motion coding.

The basic idea of our method (DCC) is to record the local curvature of the motion from the point of view of an observer traveling on the curve. The observer 
takes unit-size steps. Therefore, the next step on the curve is a change in direction toward left, right, up, down or continuing straight. We define a vocabulary for each of these changes (a code between 0 and 4) (Fig. 3).
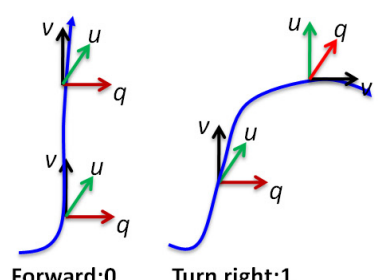

Turn right:1
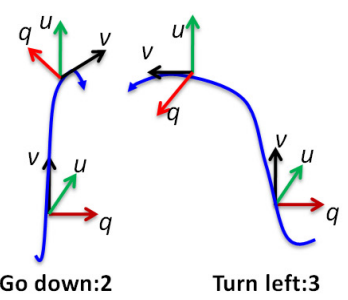
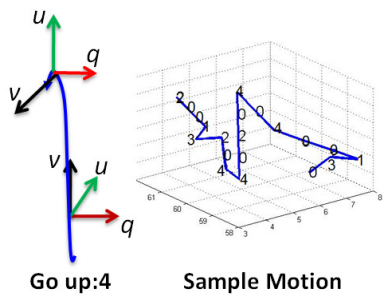

Fig. 3. DCC vocabulary and changes in direction of the attached coordinate system

The DCC then traces changes of the orthonormal 3D coordinate system attached to the observer. The local coordinate system attached to the curve $(r)$ at the step $(s)$ is defined by its three orthonormal basis vectors: the unit tangent vector $\left(v^{s}\right)$, the normal unit vector $\left(u^{s}\right)$, and binormal unit vector $\left(q^{s}\right)$. At each step $s$, the coordinate system is updated using the following equations:

$$
\begin{aligned}
& v^{s}=\left(\|d r / d s\|^{-1}\right) \frac{d r}{d s} \\
& u^{s}=u^{s-1} \text { if } c^{s-1}=0, \quad \text { otherwise } u^{s}=v^{s-1} \\
& q^{s}=\left(\left\|u^{s} \times v^{s}\right\|^{-1}\right)\left(u^{s} \times v^{s}\right)
\end{aligned}
$$

where $c^{s}$ is the code representing the tendency of the motion toward one of the 5 possible directions and is updated by projecting current $v^{s}$ onto the previous orthonormal basis $(\mathrm{x})$.

$$
c^{s}=\underset{\mathrm{i}}{\operatorname{argmax}}\left\{v^{s} \cdot\left[\mathrm{x}_{\mathrm{i}}\right]\right\}, \quad \mathrm{x}=\left[v^{s-1}, q^{s-1}, u^{s-1},-q^{s-1},-u^{s-1}\right]
$$

One advantage of the DCC is its flexibility in adding more vocabulary to the coding system, by simply adding new unit vectors in the projection function, e.g. instead of coding to four orthogonal directions we can refine the coding by adding 10 more vectors (a pairwise summation of each two projection units) to account for 45-degree changes of rotation.

Due to local interpretation of the curve, the strings generated from DCC are rotation and translation-invariant. Therefore, DCC-based skill evaluation methods are independent of the surgical site setup or the tracker reference point, whereas Cartesian-based models are sensitive to the location of the tracker and thus require registration with the tracker coordinate systems.

In this paper, from each trial's DCC string, we extract a 5-element feature vector, which is simply the normalized coding vocabulary histogram of that string. Using this feature vector, we use the same SVM configuration as described for SPP for the training and scoring of the surgical trials. We also augment the SPP feature vector with that of the DCC and explore its performance in skill evaluation. 


\section{Evaluation and Results}

We evaluate the performance of each of the models by measuring the similarity between their estimated scores $(\hat{S})$ for each surgical trial and their corresponding OSATS score $\left(S_{\text {OSATS }}\right)$. In the 4-level OSATS score (scores=[0:3]), skill evaluation error is defined as

$$
\text { Error rate }=\left|S_{\text {OSATS }}-\hat{S}\right| / l
$$

where $l$ is the normalization term (in our case $l=3$ ). This method credits partially correct decisions (That is for a $S_{\text {OSATS }}=3$ an estimation of $\hat{S}=2$ is a closer decision than $\hat{S}=0)$. We report the mean and standard deviation of the error rate - named OSATS Comparison Error Rate (OCER). We also include Sim the average similarity between the scores $\hat{S}$ and $S_{\text {OSATS }}$ :

$$
\operatorname{Sim}=1-\mu_{\mathrm{OCER}}
$$

A method with a high performance has close-to-zero OCER and close-to-one Sim and Area Under the Curve $(A U C)$. Fig 4 visualizes the results for the winner methods and a view from final location of the tool after hitting the goal in the presence of deformed tissue (visualization with VTK toolkit).

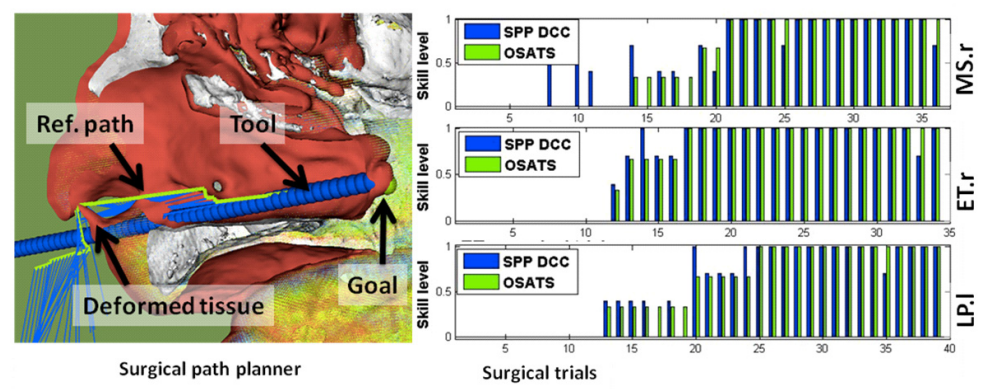

Fig. 4. (Left) Lateral clipped view of the sinus cavity where there is no straight collision-free path for tool to touch the goal. The tool follows the SPP reference path (green line) by opening its way toward the goal and putting minimum pressure on the soft tissue (blue lines). (Right) The resulting scores of SPP+DCC method (blue) and the corresponding OSATS score (green) for each trial (sorted by OSATS value, skill level of "zero" is novice and of "one" is expert).

As previously mentioned, there are no quantitative skills assessment studies that use OSATS for objective FESS evaluation. Authors in [4] represent a self-declared skill evaluation method with accuracy of $82.5 \%$ and $77.8 \%$ for expert and novice respectively. We implement their method and evaluate it against OSATS (HMM TCE in Table 2). The task ET.r was the most difficult one and resulted in a better discrimination of the dexterity.

Comparing the average success rate (Table 2) of the different techniques via replication scores (sim) shows the SPP (avg 88\%) was better than the HMM (avg 75\%) for all the tasks. The DCC method (invariant to translation and rotation of the surgical site) accurately graded the surgical trial skill (avg 87\%) and performed similarly to 
the SPP method. The successful results of DCC show that there is a noticeable "texture" in the surgical trail, which differentiates experts from novices.

Finally, one could get the advantages of both techniques by concatenating their feature vectors (SPP+DCC) and then training the SVM classifier. In this case, we get even better classification (avg 93\%). We note that the combined classification scheme has the most favorable area under the receiver operating curve and the smallest OCER of all techniques. The ROC graph for all methods and trials are shown in Fig. 5 to aid with the comparison process.

Table 2. Skill evaluation results (similarity, OSATS comparison error, Area under curve, and 95\% confidence interval) for FESS tasks (MS.r, ET.r, and LP.1) from different methods (OSATS-based HMM [4], SPP, DCC, and their concatenation)

\begin{tabular}{|c|c|c|c|c|c|c|}
\hline & \multicolumn{4}{|c|}{ Skill Evaluation Method } \\
\hline & & & HMM TCE [4] & \multirow{5}{*}{$\begin{array}{l}\text { SPP } \\
83.60 \% \\
0.16 \pm 0.30 \\
0.87 \\
0.71 \text { to } 0.96\end{array}$} & DCC & $\mathrm{SPP}+\mathrm{DCC}$ \\
\hline \multirow{12}{*}{ 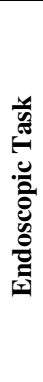 } & MS.r & Sim & \multirow{4}{*}{$\begin{array}{l}61.24 \% \\
0.38 \pm 0.31 \\
0.70 \\
0.53 \text { to } 0.84 \\
\end{array}$} & & \multirow{4}{*}{$\begin{array}{l}80.46 \% \\
0.19 \pm 0.31 \\
0.88 \\
0.73 \text { to } 0.96\end{array}$} & \multirow{4}{*}{$\begin{array}{l}87.30 \% \\
0.12 \pm 0.24 \\
0.98 \\
0.87 \text { to } 1.00\end{array}$} \\
\hline & & OCER & & & & \\
\hline & & AUC & & & & \\
\hline & & $95 \% \mathrm{CI}$ & & & & \\
\hline & \multirow[t]{4}{*}{ ET.r } & Sim & \multirow{4}{*}{$\begin{array}{l}\mathbf{7 9 . 3 5 \%} \\
0.20 \pm 0.28 \\
0.81 \\
0.65 \text { to } 0.93\end{array}$} & \multirow{4}{*}{$\begin{array}{l}\mathbf{9 5 . 1 9 \%} \\
0.04 \pm 0.13 \\
0.95 \\
0.81 \text { to } 0.99\end{array}$} & \multirow{4}{*}{$\begin{array}{l}\mathbf{9 2 . 1 5 \%} \\
0.07 \pm 0.20 \\
0.84 \\
0.67 \text { to } 0.94\end{array}$} & \multirow{4}{*}{$\begin{array}{l}97.64 \% \\
0.02 \pm 0.07 \\
0.96 \\
0.833 \text { to } 0.99\end{array}$} \\
\hline & & OCER & & & & \\
\hline & & AUC & & & & \\
\hline & & $95 \% \mathrm{CI}$ & & & & \\
\hline & \multirow[t]{4}{*}{ LP .l } & Sim & \multirow{4}{*}{$\begin{array}{l}75.38 \% \\
0.24 \pm 0.26 \\
0.81 \\
0.65 \text { to } 0.91\end{array}$} & \multirow{4}{*}{$\begin{array}{l}\mathbf{8 5 . 2 9 \%} \\
0.14 \pm 0.26 \\
0.86 \\
0.71 \text { to } 0.95\end{array}$} & \multirow{4}{*}{$\begin{array}{l}\mathbf{8 8 . 3 7 \%} \\
0.11 \pm 0.18 \\
0.86 \\
0.71 \text { to } 0.95\end{array}$} & \multirow{4}{*}{$\begin{array}{l}94.70 \% \\
0.05 \pm 0.12 \\
0.95 \\
0.83 \text { to } 0.99\end{array}$} \\
\hline & & OCER & & & & \\
\hline & & $\mathrm{AUC}$ & & & & \\
\hline & & $95 \% \mathrm{CI}$ & & & & \\
\hline
\end{tabular}

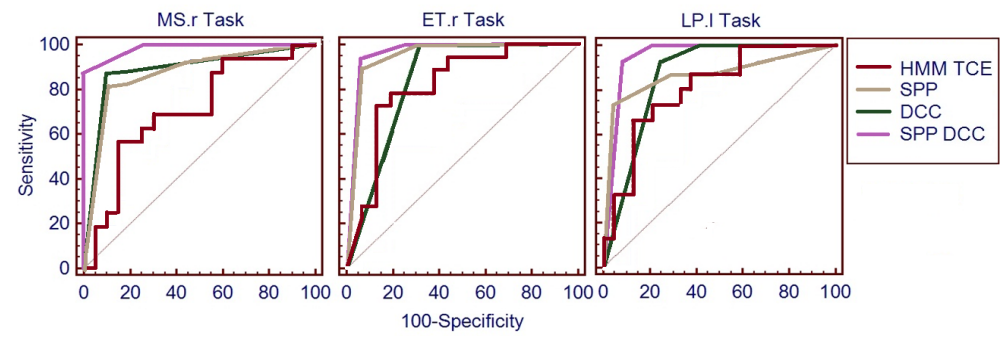

Fig. 5. ROC curves for different skill evaluation methods

\section{Conclusion}

In this paper, we introduced an automated skill assessment technique for Functional Endoscopic Sinus Surgeries (FESS). First, we showed that OSATS grading - the ground truth for evaluation of the resident surgical skills- is not consistent with the overall self-declared scores. Then, we introduced an OSATS-based automated skill evaluation technique. We believe that there is an optimal path for performing a FESS task which is close to what an expert surgeon would do in an ideal setup. The optimal path can be flexibly defined based on the faculty surgeon's approach in performing the surgery. Typically there is no collision-free path for FESS tasks, so here the refer- 
ence path is optimized for the shortest distance to goal and least collision with the soft tissue. To explore the idea, we built a Surgical Path Planner (SPP) where the obstacles were extracted from CT. We then compared the SPP path to the one performed by the surgeons. Our results show the surgeons with higher OSATS are closer to the SPP path than the ones with lower scores. We then improved the skill assessment technique by translating the Cartesian-space motions to a coded domain using Descriptive Curve Coding (DCC). The new domain is rotation and translation-invariant and represents the "texture" of the motion. We showed that this representation amplifies the skill-related structures.

As the future work, we would like to investigate the success rate of the grammarbased DCC methods. For example, a substring with two sequential 4's might represents "retraction" which could be evaluated as a novice behavior. A sequence of zeros shows a clean and straight insertion. A substring of interleaved 2's and 3's reveals the jitter in the surgeon's hand.

Acknowledgments. This work was funded by NSF CDI-0941362. Any opinions, findings, conclusions or recommendations expressed in this material are those of the authors and do not necessarily reflect the views of the National Science Foundation.

\section{References}

1. Martin, J.A., Regehr, G., Reznick, R., MacRae, H., Murnaghan, J., Hutchison, C., Brown, M.: Objective Structured Assessment of Technical Skill for Surgical Residents. British Journal of Surgery 84, 273-278 (1997)

2. Reiley, C.E., Lin, H.C., Yuh, D.D., Hager, G.D.: A Review of Methods for Objective Surgical Skill Evaluation. Surgical Endoscopy 25, 356-366 (2011)

3. Padoy, N., Blum, T., Ahmadi, A., Feußner, H., Berger, M.O., Navab, N.: Statistical Modeling and Recognition of Surgical Workflow. J. Med. Image Analysis 16, 632-641 (2010)

4. Ahmidi, N., Hager, G.D., Ishii, L., Fichtinger, G., Gallia, G.L., Ishii, M.: Surgical Task and Skill Classification from Eye Tracking and Tool Motion in Minimally Invasive Surgery. In: Jiang, T., Navab, N., Pluim, J.P.W., Viergever, M.A. (eds.) MICCAI 2010, Part III. LNCS, vol. 6363, pp. 295-302. Springer, Heidelberg (2010)

5. Reiley, C.E., Hager, G.D.: Task versus Subtask Surgical Skill Evaluation of Robotic Minimally Invasive Surgery. In: Yang, G.-Z., Hawkes, D., Rueckert, D., Noble, A., Taylor, C. (eds.) MICCAI 2009, Part I. LNCS, vol. 5761, pp. 435-442. Springer, Heidelberg (2009)

6. Gayle, R., Segars, P., Lin, M.C., Manocha, D.: Path planning for deformable robots in complex environments. In: Robotics Systems and Science (2005)

7. Rilk, M., Wahl, F.M., Eichhorn, K.W.G., Wagner, I., Bootz, F.: Path Planning for RobotGuided Endoscopes in Deformable Environments. J. Advances in Robotic, 263-274 (2009)

8. Nain, D., Haker, S., Kikinis, R., Eric, W., Grimson, L.: An Interactive Virtual Endoscopy Tool. In: IMIVA workshop, MICCAI (2001)

9. Choset, H., Lynch, K.M., Hutchinson, S., Kantor, G., Burgard, W., Kavraki, L.E., Thrun, S.: Principles of Robot Motion: Theory, Algorithms, and Implementations. MIT Press (2005)

10. Weisstein, E.W.: CRC encyclopedia of mathematics. CRC Press, Taylor \& Francis (2009)

11. Bribiesca, E., Aguilar, W.: A Measure of Shape Dissimilarity for 3D Curves. Int. J. Contemp. Math. Sciences 1, 727-751 (2006) 